\title{
Striving for perfection
}

Dawn S. Hui, MD, and Richard Lee, MD, MBA

From the Center for Comprehensive Cardiovascular Care, Saint Louis University, Saint Louis, Mo.

Authors have nothing to disclose with regard to commercial support.

Received for publication May 1, 2018; revisions received May 1, 2018; accepted for publication May 2, 2018; available ahead of print June 19, 2018

Address for reprints: Richard Lee, MD, MBA, 3635 Vista Ave, DT 13th Fl, Saint Louis, MO 63110 (E-mail: rick. lee@health.slu.edu).

J Thorac Cardiovasc Surg 2018;156:1408-9

$0022-5223 / \$ 36.00$

Copyright $\subset 2018$ Published by Elsevier Inc. on behalf of The American Association for Thoracic Surgery

https://doi.org/10.1016/j.jtcvs.2018.05.015

What is the perfect coronary artery bypass graft (CABG) operation? Ideally, it is an operation that protects against future cardiovascular events by maintaining graft patency and freedom from arteriosclerosis and confers a survival benefit. What is the perfect comparative effectiveness study? The ideal study design would observe outcomes for the same patients under different conditions of exposure while keeping all other conditions (including time-related conditions) constant-what is called the counterfactual ideal. Although this design is impossible to achieve, cardiac surgeons strive daily to perform the perfect CABG procedure.

Kim and colleagues ${ }^{1}$ present an extended analysis of the Saphenous Vein versus Right Internal Thoracic Artery as a Y-Composite graft (SAVE RITA) trial, which was a randomized study on left internal thoracic artery (ITA)based composite grafts, comparing the right ITA (RITA) to saphenous vein (SV) in a $Y$ configuration. Using an $8 \%$ noninferiority margin, the authors found that SV grafts were noninferior in angiographic patency at 5 years and noninferior in survival or major adverse cardiovascular and cerebrovascular events (MACCE) at 5 and 8 years. The authors have demonstrated impressive patency results, with graft occlusion rates of only $3.6 \%$ at 5 years. However, in terms of study design, readers should be cautious of interpreting the results at face value. Knowing the history of the SAVE RITA study is instrumental. As originally designed, ${ }^{2}$ the SAVE RITA power calculation was based on 1 -year patency rates of $93 \%$ for SV and $95 \%$ for RITA. In their earlier published results, 1-year angiograms were performed in $99 \%$ of patients. In the current study, ${ }^{1}$ $15 \%$ did not undergo coronary evaluation, and $46.8 \%$ were evaluated by multidetector computed tomography rather than the gold-standard angiography. Again, the authors should be commended for patency rates that exceeded those specified for the power calculation. Yet, how we should now judge the adequacy of the sample size at 5 years is in doubt, especially in light of an overall combined loss to follow-up or protocol violation rate of $17 \%$. Additionally, there may be confounders that were not addressed in the study, including lack of target vessel

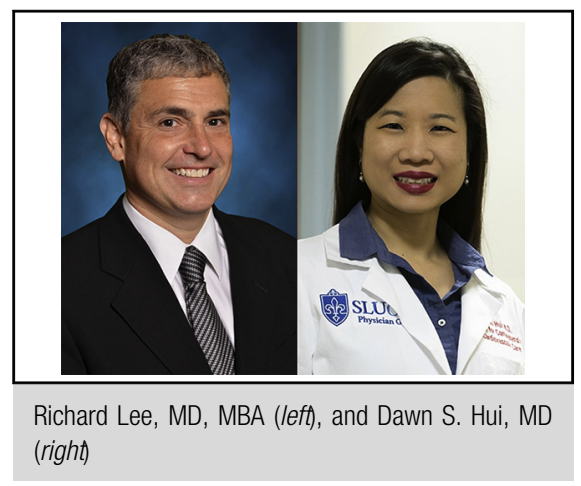

\section{Central Message}

Randomized studies on arterial revascularization are rare. Preserving follow-up and longterm angiographic evaluation are challenges to interpreting study results.

See Article page 1424.

stenosis percentage and the differential rate in third limb conduit use.

Despite the limitations of the study and the potential for a spurious conclusion, the results suggest that SV patency rates can be significantly improved upon. Although there has been universal acknowledgment that arterial grafts are superior to venous grafts, the benefit of bilateral ITA has typically been demonstrated in the longer term. The relatively flat rate of bilateral ITA use at $4 \%$ to $5 \%$ in the United States betrays an unwillingness to adoption in practice. In light of this, perhaps the value of this study is in encouraging improvements in SV patency. Determining whether patency is related to the composite left ITAbased nature, the absence of manual dilation, minimization of manipulation, anastomotic technique, or selection of patients and targets will require a multi-institutional study.

Finally, the study by Kim and colleagues ${ }^{1}$ demonstrates the challenges in conducting randomized trials in the field of surgical coronary revascularization. The timing of angiographic findings does not equate to the timing of physiologic occlusion, nor of meaningful clinical benefit. With excellent surgical results, the timeline to a benefit difference becomes longer, risking further and further loss to follow-up, or non-MACCE clinical events that erode statistical power. The 5-year results of the Arterial Revascularization Trial continued to demonstrate no statistically significant difference between bilateral ITA and SV graft. $^{3}$ A randomized study of 
in situ versus $Y$ grafting configurations of bilateral ITA demonstrated no angiographic difference at 3 years and no survival difference at 7 years. ${ }^{4}$ However, at 7 years there were significant differences in MACCE, myocardial infarction, and percutaneous coronary intervention, revascularization, and stroke rates. Perhaps all we need is a little patience. The upcoming 10-year Arterial Revascularization Trial results and perhaps longer-term follow-up of the study by Kim and colleagues ${ }^{1}$ will surely be worth the wait in the ongoing quest for the perfect CABG procedure.

\section{References}

1. Kim M-S, Hwang HY, Kim JS, Oh SJ, Jang M-J, Kim K-B. Saphenous vein versu right internal thoracic artery as a Y-composite graft: five-year angiographic and clinical results of a randomized trial. J Thorac Cardiovasc Surg. 2018;156: 1424-33.e1.

2. Kim K-B, Hwang HY, Hahn S, Kim JS, Oh SJ. A randomized comparison of the Saphenous Vein versus Right Internal Thoracic Artery as a $Y$-Composite graft (SAVE RITA) trial: one-year angiographic results and mid-term clinical outcomes. J Thorac Cardiovasc Surg. 2014;148:901-8.

3. Taggart DP, Altman DG, Gray AM, Lees B. Randomized trial of bilateral versus single internal-thoracic-artery grafts. N Engl J Med. 2016;375:2540-9.

4. Glineur D, Boodhwani M, Hanet C, de Kerchove L, Navarra E, Astarci P, et al. Bilateral internal thoracic artery configuration for coronary artery bypass surgery: a prospective randomized trial. Circ Cardiovasc Interv. 2016;9:e003518. 